Dieses Dokument ist eine Zweitveröffentlichung (Verlagsversion) / This is a self-archiving document (published version):

Alexander Lasch

Zur Vereinbarkeit von diskurslinguistisch motivierter Sprachgeschichtsschreibung und maschineller Sprachanalyse am Beispiel des „Islamismus“-Diskurses

Erstveröffentlichung in / First published in:

Jahrbuch für Germanistische Sprachgeschichte. 2014, 5 (1), S. 231 - 249. De Gruyter. ISSN: 1869-7046.

DOI: https://doi.org/10.1515/jbgsg-2014-0016

Diese Version ist verfügbar / This version is available on:

https://nbn-resolving.org/urn:nbn:de:bsz:14-qucosa2-748614 


\section{Zur Vereinbarkeit von diskurslinguistisch motivierter Sprachgeschichtsschreibung und maschineller Sprachanalyse am Beispiel des „Islamismus“-Diskurses}

Aktuelle Sprachgeschichtsforschung arbeitet sprachgebrauchsbasiert und korpusgestützt; die Zeiten, in denen Sprachformen erschlossen oder geglättete Editionen mittelhochdeutscher Überlieferung erarbeitet wurden, scheinen vorbei zu sein. Zum zweiten haben in den letzten 30 Jahren sprachexterne Aspekte wesentlich an Gewicht gewonnen: $\mathrm{Zu}$ denken ist an alle Ansätze einer pragmatischen und später kulturwissenschaftlich interessierten Sprachgeschichtsschreibung, die die Kontexte kommunikativer Ereignisse berücksichtigen. So ist verständlich, dass die Diskurslinguistik nach dem Zuschnitt Busses seit 20 Jahren in der Sprachgeschichtsforschung starken Widerhall findet und als Theorie- und Methodenangebot aufgenommen wurde, um die Spezifik komplexer kommunikativer Ereignisse zu beschreiben.

In den letzten Jahren gewinnen außerdem maschinelle Analysen auf der Basis großer maschinenlesbarer Korpora zunehmend an Bedeutung im Bereich der Linguistik. Die Sprachgeschichtsforschung geht (noch zaghaft) ins digitale Zeitalter, vor der Nutzung maschinenlesbarer Korpora hat sie auf weiten Strecken noch gehörigen Respekt.

Das hat neben anderen auch möglicherweise diese zwei Gründe: Zum einen liegt der Diskurslinguistik (und damit diskurslinguistischen sprachhistorischen Arbeiten) ein Korpusbegriff zu Grunde, der mit dem Korpusbegriff der Korpuslinguistik nicht ohne Weiteres vereinbar zu sein scheint. Zum anderen - und das ist forschungspraktisch der ausschlaggebende Punkt - müssen maschinenlesbare Korpora standardisiert und zuverlässig annotiert sein, um belastbare Ergebnisse liefern zu können. Dazu müssten alle Wortvorkommen in historischen Texten nach grammatischen Informationen und ihrer neuhochdeutschen Entsprechung annotiert werden. Das ist in Bezug auf historische Quellen jenseits des standardisierten 19. Jahrhunderts ein nicht zu unterschätzendes Problem und könnte beim jetzigen Stand der Technik zum großen Teil nur von Hand erfolgen.

Dass sich der Aufwand dennoch lohnt, lässt sich an ausgewählten Beispielen aber schon heute zeigen. Im vorliegenden Beitrag soll der Versuch unternommen werden, auf der Basis eines maschinenlesbaren Korpus signifikante sprachliche Muster (Konstruktionen) herauszuarbeiten, die im „Islamismus“-Diskurs Argu- 
mentationslogiken unterstützen. Dazu werde ich zunächst kurz die Ansätze der Diskurslinguistik (vgl. Abschnitt 1.1) und der Konstruktionsgrammatik (vgl. Abschnitt 1.2) in gebotener Kürze vorstellen, aufeinander beziehen und mich den eingangs aufgeworfenen Fragen nach den je unterschiedlichen Verständnissen von Korpora (vgl. Abschnitt 1.3) zuwenden. In einem zweiten Schritt (Abschnitt 2) setze ich mich mit dem „Islamismus“-Diskurs und der so genannten „Konstruktion der Kontravalenz“ auseinander, um zu zeigen, welche Erkenntnisse aus der Analyse sprachlicher Muster als Konstruktionen historische qualitative Studien bereichern können.

\section{Theoretische Vorüberlegungen}

\subsection{Diskurslinguistische Prämissen}

Die Diskurssemantik nach Dietrich Busse (1987) sowie Busse / Teubert (1994, Nachdruck 2013) ist einer der zentralen Ansätze, die sich mit dem Zusammenhang von Sprache und Wissen auseinandersetzen. Mit dem Begriff des Diskurses nach Foucault kamen zum einen Wissensformationen in den Blick, die in Sprache sedimentierten. Zum anderen und über Foucault ausgreifend ließen sich mit dem Diskursbegriff die Bedingungen beschreiben, unter denen Sprache Wissensformationen etabliere und stabilisiere: Im Mittelpunkt stehen in den Analysen diskurssemantische Grundfiguren (vgl. exemplarisch Busse 1997 oder 2000), die sich aus sprachlichen (und nichtsprachlichen) Einheiten unterschiedlicher Komplexität rekonstruieren lassen. Um diese adäquat beschreiben zu können, werden Diskurse als immer bereits kontextualisiert charakterisiert (vgl. Busse 2007, 82):

„[...] Kontextualisierung ist eigentlich und von allem Anfang ein epistomologischer (kognitiver) Begriff. Kontexte [...] sind keine objektiven Daten, sie sind nicht gegeben, sondern müssen gesucht, gefunden und hergestellt werden.“ (Busse 2007, 102 und vgl. weiter 2008).

Forschungspraktisch greift man zur Erschließung von Diskursen auf alle methodischen Ansätze zurück, die einer interpretierenden Linguistik zur Verfügung stehen. ${ }^{1}$ Dazu gehört auch die Framesemantik, die in den letzten Jahren an Relevanz für die Diskurslinguistik gewinnt (vgl. zuletzt Busse 2012). In diesem Zusam-

1 Den Versuch einer Übersicht und Zusammenstellung der üblichen Methoden geben Spitzmüller / Warnke 2011. 
menhang wird ein Modell virulent, dass 1985 bereits durch Peter von Polenz (2008) in seiner Satzsemantik vorgestellt wurde. Von Polenz postulierte basierend auf Fillmores Kasusgrammatik eine „Inhaltsgrammatik“, die der Bedeutung sprachlicher Einheiten starkes Gewicht gab und für aktuelle diskurslinguistische wie konstruktionsgrammatische Studien von großem Wert ist. Den Prämissen einer korpusgestützten Konstruktionsgrammatik kommt schließlich eine weitere Entwicklung entgegen, die mit den Arbeiten von Hermanns $\mathrm{zu}$ verbinden ist. Er forderte früh $(1995,71)$ eine stärkere Orientierung sprachwissenschaftlicher Studien an seriellen und usuellen Quellen, um u.a. Mentalitäten als (kollektive) Wissensformen in Diskursen $\mathrm{zu}$ analysieren. In Nachfolge Coserius (vgl. 1974, 247) und Schlieben-Langes (vgl. etwa 1983, 465) machte Hermanns das Konzept der „Annales“ stark. ${ }^{2}$ Denn die

„Analysen eines bestimmten Phänomens [ergeben] oftmals über lange Zeiten hinweg lange Reihen von gleichartigen Belegen, also Hinweise auf die Stabilität dieser Phänomene auf allen Ebenen des Sprachgebrauchs.“ (Mattheier 1995, 13).

Diese Sicht auf die Entwicklung stabiler bzw. innovativer sprachlicher Oberflächenphänomene und Diskurse bringt zwangsläufig eine Neubewertung des Korpus-Verständnisses der Diskurslinguistik mit sich. Dieser wenden wir uns nach einem Blick auf die konstruktionsgrammatischen Grundannahmen zu.

\subsection{Konstruktionsgrammatische Grundannahmen}

Eine „Konstruktion“ ist nach Goldberg (1995 und 2006) sowie Ziem / Lasch (2013) eine sprachliche Einheit, die als Form-Bedeutungs-Paar Ergebnis von Sprachgebrauchs- und Konventionalisierungsprozessen ist. Diese Einheiten sind nicht vollständig hinsichtlich ihrer Bedeutung vorhersagbar bzw. usuell und seriell nachweisbar. Konstruktionen entstehen aus Gebrauch, sind kognitive Repräsentationen und in einem ,Konstruktikon', einem Netzwerk, geordnet. ${ }^{3}$ Konzepte wie diese werden schon länger diskutiert: So warb Feilke bereits 1996 für eine „Sprache als soziale Gestalt“ und diskutierte die Konventionalisierungsgrade

2 Vgl. Honegger 1977, 7-4. - Zur „Annales“ siehe auch Lasch 2005, 105 mit Anm. 616 sowie 2013 und auch Spitzmüller / Warnke 2011, 85-87.

3 Vgl. dazu meist ausgehend Goldberg (und der Bestimmung des construct-i-cons in Goldberg 2003, 219) sowie Ziem / Lasch 2013 und darüber hinaus die Artikel, die im Band Lasch / Ziem (2014) dem Konstruktikon besondere Aufmerksamkeit widmen: Hervorzuheben sind die Beiträge Boas 2014, Bücker 2014, Lasch 2014, Rostila 2014 sowie Ziem $2014 a$ und b. 
unterschiedlich idiomatisierter Einheiten vor dem Hintergrund der Überwindung der Trennung von Lexikon und Grammatik (vgl. besonders deutlich Feilke 1996, 211 ff. und 239). Auch die kognitive Linguistik, z. B. die Cognitive Construction Grammar nach Lakoff (vgl. Ziem / Lasch 2013, 39-41), arbeitet an sprachlichen Phänomenen, die auf kognitive Gestalten in genannter Art hindeuten.

Konstruktionen sind Formen, vielleicht die Formen, von Sprachwissen. Ihre Realisierungen lassen es $\mathrm{zu}$, auf bewusste (surface frames) und unbewusste (aber meist nicht explizierte) und gelernte Verwendungskontexte, Gebrauchsregeln und Aussageabsichten (deep seated frames) zu schließen. ${ }^{4}$ Sie geben als sprachliche Muster einen Hinweis darauf, wie zu welcher Zeit in welcher Art zu wem über welches Thema gesprochen wurde oder eben gerade nicht. Sie sind die kleinsten Einheiten unterhalb der Ebene der bisher durch die Diskurslinguistik anvisierten „Aussagenkomplexe“, deren Bedeutung sich für eine Diskursanalyse produktiv machen lässt. Gebrauchshäufigkeiten geben Hinweise auf den diskursspezifischen Gebrauch von Konstruktionen, besondere Prägungen ihrer in diesen Kontexten aktualisierten Bedeutungen zeigen semantische Spezifizierungen von Konstruktionen an, die sich für einen Kontext als typisch erweisen können. Mit anderen Worten: Die Analyse von Konstruktionen und Realisierungen von Konstruktionen als konventionalisierten sprachlichen Einheiten mit dem Blick auf ihre Bedeutung unter Berücksichtigung ihrer Kontextualisierung verfolgt ähnliche Ziele wie die Diskurslinguistik und damit eine diskurslinguistisch motivierte neuere Sprachgeschichte.

\subsection{Diskussion der Konzepte von Korpora}

Unter dem „,virtuellen Korpus“ fassten Busse und Teubert alle Kommunikate, die sich mit dem als Forschungsgegenstand gewählten Thema befassen und semantische Beziehungen aufweisen - den „Diskurs“ (Busse / Teuber 2013, 16). Die Kommunikate stehen in einem inhaltlichen Zusammenhang und genügen unterschiedlichen forschungspraktischen Bedingungen (Zeitraum, Areal, Gesellschaftsausschnitt, Texttypik, Kommunikationsbereich etc.) (vgl. Busse / Teubert 2013, 17). Aus dem „virtuellen Korpus“ wird das „konkrete Korpus“ der Untersuchung herausgearbeitet, das die Basis einer Diskursanalyse bildet: „Konkrete

4 Zur Einführung in die kognitionslinguistische Theorie der Metaphern vgl. Lakoff / Wehling 2009 (in Bezug auf surface frames und deep seated frames besonders S. 73-87). Die Übersetzung basiert auf Lakoff 2002, der zweiten Aufl. eines Textes von 1996. Vgl. weiter Lakoff / Johnson 1980 (Argument is war) und Lakoff 1987. Zur Diskussion des Begriffes Frame, der in unterschiedlichen Schulen je unterschiedlich verwendet wird, vgl. zuletzt Busse 2012 sowie Ziem 2008 und 2014b. 
(d. h. einer diskurssemantischen Untersuchung zugrunde liegende) Textkorpora sind Teilmengen der jeweiligen Diskurse“ (Busse / Teubert 2013, 17), die nicht auf der Basis quantitativer (Repräsentativität) sondern qualitativer Kriterien („Relevanzkriterien“) ermittelt werden. Das „konkrete Korpus“ ist bereits erste Interpretationsleistung: Es wird durch den Wissenschaftler konstituiert, der sich durch sein Interesse am Material leiten lässt und vor allem diskursveränderungsrelevante Texte aus dem „virtuellen“ für das „konkrete Korpus“ zusammenstellt (Busse / Teubert 2003, 17). Das „konkrete Korpus“ ist ein Ausschnitt des Diskurses. Mit der Forderung nach der Analyse des Seriellen und Usuellen (exempl. Hermanns 1995, Mattheier 1995, Felder / Müller / Vogel 2012, Scharloth / Eugster / Bubenhofer 2013 und Lasch 2013) für diskurslinguistische Analysen ist seit einigen Jahren zu beobachten, dass sich das Korpusverständnis der Diskurslinguistik nachhaltig ändert. Präziser muss man sagen, dass mit der Etablierung maschineller Analysen das „virtuelle Korpus“ für diskurslinguistische Analysen stärker in den Blick rückte, es kann zur Genese von Hypothesen Gegenstand maschineller Analysen werden.

Gebrauchsbasierte Ansätze der Konstruktionsgrammatik setzen immer stärker auf maschinelle Analysen und damit auf eben solche „virtuellen Korpora“. Sie greifen aber meist noch darüber hinaus, da sie nicht nur an einem ,Thema' interessiert sind, sondern prinzipiell am (varietätengebundenen) Sprachgebrauch zu einem gegebenen Zeitpunkt. Das hat für die Diskurslinguistik einen besonderen Reiz, da so die Möglichkeit besteht, die Typik eines sprachlichen Musters, einer Konstruktion, für ein „,virtuelles Korpus“ im Vergleich zu anderen „virtuellen Korpora“ herauszuarbeiten. So erhält man unter Umständen Hinweise darauf, auf welche besonderen sprachlichen Muster diskursdominante Aussagenkomplexe aufbauen. Das war der Diskurslinguistik und der diskurslinguistisch motivierten Sprachgeschichte bisher in dieser Form noch nicht möglich.

Mit einem unterschiedlichen Korpusverständnis unterscheiden sich die anvisierten Gegenstände der Diskurslinguistik und der Konstruktionsgrammatik. Allerdings können wie gesehen die Konzepte von Korpora so auf einander bezogen werden, dass die korpuslinguistisch gestützte Konstruktionsgrammatik zum Hypothesengenerator einer diskurslinguistisch motivierten Sprachgeschichte werden kann. 


\section{Die „Konstruktion der Kontravalenz“ im „Islamismus“-Diskurs}

Einige der Konsequenzen der bisher angestellten theoretischen Überlegungen möchte ich nun an einer kleinen Beispielanalyse aufzeigen. Wie eingangs erwähnt, sind für die Erschließung „virtueller Korpora“ durch maschinelle Analyse Annotationen von hoher Qualität notwendig, die eine standardisierte Übertragung ins Neuhochdeutsche mit einschließen. Ohne diese Standardisierung sind Entwicklungslinien von Sprache anhand sprachlicher Muster, Konstruktionen, kaum plausibel nachzuvollziehen.

Korpora als Datensammlungen, die diesem Anspruch genügen, gibt es bis zum jetzigen Zeitpunkt nicht; sie wären die Grundlage „virtueller Korpora“, die in Bezug auf ein bestimmtes Thema zusammengestellt werden. Aus diesem Grund ist es im Moment nur möglich, die Leistungsfähigkeit eines solchen Zugriffs über einen Umweg anzudeuten, was hier geschehen soll.

Dazu wählen wir die andere Blickrichtung: Wir erstellen (1) erst auf der Basis einer Schlagwortanalyse ein „konkretes Korpus“ aus dem „virtuellen Korpus“ zum „Islamismus“-Diskurs. Dieses „konkrete Korpus“ analysieren wir (2) hinsichtlich auffälliger sprachlicher Muster, die wir dann in anderen ,virtuellen Korpora“ weiterverfolgen können im Rahmen einer qualitativen Analyse. So können wir Aussagen darüber machen, ob das Auftreten bzw. Ausbleiben spezifischer sprachlicher Muster, Konstruktionen, Indiz für sprachlich sedimentiertes Wissen ist, das in einem thematisch zentrierten Diskurs (,virtuelles Korpus“) auf spezifische Weise prozessiert wird. Man würde z. B. in unserem Falle sagen können, dass wir bestimmte sprachliche Einheiten, Konstruktionen, und das Schlagwort Islamismus als surface frames auffassen, die möglicherweise im Umfeld spezifischer deep seated frames als kognitive Routinen verwendet werden, diese u.a. aktivieren und aktiv halten.

Ausgangspunkt unserer Überlegung ist die Beobachtung, dass der Begriff Islamismus in der medialen Öffentlichkeit der Gegenwart synonym für eine Geisteshaltung oder Doktrin verwendet zu werden scheint, die zu gewaltsamen Handlungen gegenüber Anders- bzw. Nichtgläubigen im Namen des als gerecht konzeptualisierten Islams aufruft und dabei vornehmlich Gesellschaften im ,Westen“ im Blick hat. In diesem Sinne wird er in den deutschsprachigen öffentlichen Diskursen ab Mitte der 1980er Jahre verwendet, dabei ist er viel älter, wie ein erster Blick auf eine, zugegeben, sehr heterogene Datenbasis bestätigt: ${ }^{5}$

5 Die mit dem nGram Viewer von Google erhobenen Daten halten der wissenschaftlichen Prüfung nicht stand (Transparenz, Dokumentation). Für einen ersten Blick auf die Entwicklung be- 


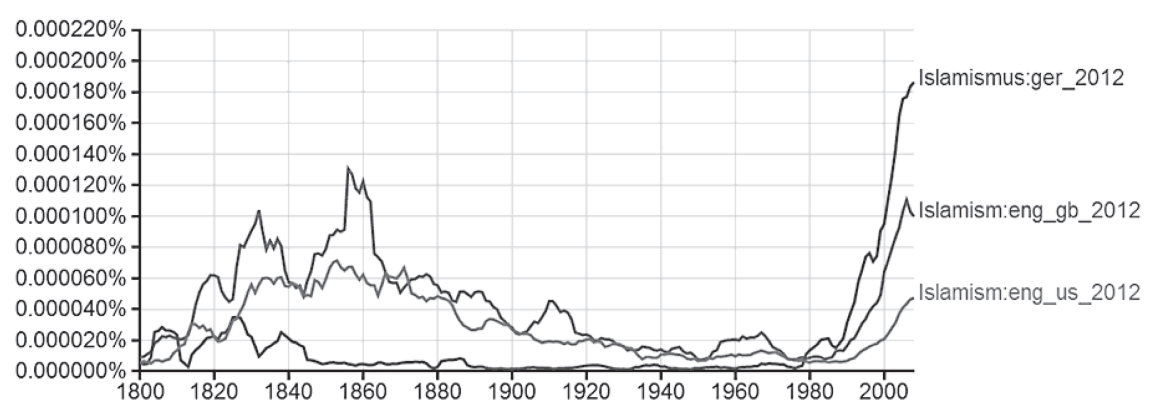

Abb. 1: Islamism, Islamismus in den englisch- und deutschsprachigen Korpora bei Google Books.

Für den ,Westen“ ist der Anschlag 1983 in Beirut wohl das maßgebliche Ereignis, ${ }^{6}$ das man zur Erklärung des rasanten Frequenzanstiegs hinzuziehen könnte. Für Deutschland und den öffentlichen Diskurs sind außerdem das Attentat München 1972 und vor allem die Entführung der Landshut 1977 im so genannten „Deutschen Herbst“ prägend (vgl. u.a. die Aufarbeitung durch Aust 2008). Diese erste Beobachtung lässt sich auch in systematisch zusammengestellten Korpora bestätigen wie im (nicht öffentlich zugänglichen) SPIEGEL-Korpus, das seit 1947 alle Ausgaben und damit knapp 238 Millionen Wortformen (in über 300.000 Texten) in einem annotierten Korpus umfasst und hier verwendet wird.

Anhand dieser ersten Beobachtungen, dass der Begriff Islamismus im 19. Jahrhundert nachgewiesen werden kann, aber erst seit 1980 hochfrequent verwendet wurde, kann eine Arbeitshypothese für eine diskurslinguistische sprachhistorische Studie formuliert werden: Die Konzeptualisierung des Begriffs Islamismus (und seinen Ableitungen Islamist sowie islamistisch) ist in den öffentlich medialen Diskursen (und die europäischen Nationalsprachen übergreifend) weitestgehend stabil, Frequenzunterschiede lassen sich durch außersprachliche Ereignisse erklären. Die Antithese lautet: Erst im Zuge mehrerer Anschlagsserien in der ,westlichen' Welt wurde der Begriff Islamismus (und seine Ableitungen Islamist und islamistisch) zum Schlagwort für den gewaltsamen, religiös motivierten Kampf gegenüber Anders- bzw. Nichtgläubigen im Namen des Islams. Der Frequenzanstieg ist sowohl Reflex auf außersprachliche Ereignisse als auch Indiz für die Neuprägung des Begriffs - es ist diese Janusgesichtigkeit eines Begriffes,

stimmter sprachlicher Phänomene (vor allem im 19. Jh.) ist er nützliches Hilfsmittel. Zur genauen Abfrage: http://goo.gl/17Jxqv, Stand: 13.07.2014.

6 Vgl. „Report of the DOD Commission on Beirut International Airport Terrorist Act, October 23, 1983“, http://goo.gl/vK87GO, Stand: 13.07.2014. 
die Busse im Blick hat, wenn er Foucaults Diskursbegriff um eine pragmatische Dimension erweitert.

Für die folgenden Überlegungen seien einige Beispiele zusammengestellt für die Verwendung des Begriffs Islamismus (und seine Ableitungen) im SPIEGELKorpus vor (1) und nach 1983 (2-4, Hervorhebung von mir, A.L.):

(1) Gegenstelle für den Report war in der Dokumentation („SPIEGEL-Archiv“) Sibylla Schuster-Walser, promovierte Islamistin, Turkologin und Indogermanistin, aber dennoch auch hier sachverständig: Sie stammt aus Basel. (SPIEGEL-001)

(2) Kritische Intellektuelle wie der Schriftsteller Mimouni glauben zwar, daß die Islamisten nur deshalb so einflußreich werden konnten, weil die FLN keine Alternative zuließ. Aber sicher scheint, daß die Frommen, fänden heute wirklich freie Wahlen statt, ein gutes Drittel der Stimmen auf sich vereinen könnten. (SPIEGEL-002)

(3) Und die Programme von Ecevit und Bahçeli decken sich mit den Machtstaat-Visionen der Generäle: Verteidigung der säkularen Republik gegen die Islamisten und keinerlei politischen Spielraum für die Kurden. (Spiegel-003)

(4) Kaplan gilt als Sinnbild des fundamentalistischen Eiferers, und der Ärger mit ihm ist auch noch nicht ausgestanden. Drei Dutzend Anhänger des Islamistenführers griffen die Polizisten gleich nach dessen Verhaftung an, traktierten sie mit Latten und Schaufeln. 14 Beamte gingen verletzt zu Boden. (Spiegel-004)

Bereits an diesen Beispielen wird offensichtlich, dass die Antithese bestätigt wird: Das Schlagwort Islamismus (und seine Ableitungen) wird in den 1980ern neu geprägt. Bis zur Neuprägung wird es verwendet als Begriff für eine Religionsgemeinschaft bzw. sogar wie im Beispiel für die Bezeichnung einer Wissenschaftlerin. Der Begriff wird also verwendet analog zu dem des Christentums - und anderen Weltanschauungen und Ideologien. Das ist auch die Verwendung, die in Quellen des 19. Jahrhunderts nachgewiesen werden kann (Hervorhebung von mir, A.L.):

(5) Worin besteht der Islamismus? fragt ein Engel, der die Gestalt eines arabischen Beduinen angenommen. (Oelsner 1810, 30 f.)

(6) Wir können diese Bemerkungen nicht schließen, ohne mit einem Worte auch [d]ie Renegaten erw(hnt zu haben, worunter man bekanntlich die versteht, welche vom Judenthum und vom Christenthum zum Islamismus übertreten. (Schmid 1815, 478)

(7) Islamismus (vom arab. Islam, Friede, Heil, Glaube) ist soviel als Muhammedanismus, indem derselbe von [...] Muhammed [...] gestiftet wurde. (Krug 1833, 554) 
Das Umschlagen des Begriffs, die Neuprägung, geht in den Belegen auf den ersten Blick mit einer negativen Bewertung einher, die sich möglicherweise auch in anderen sprachlichen Mustern zeigt als ausschließlich im hochfrequenten Gebrauch eines oder mehrerer Schlagwörter (Hervorhebung von mir, A.L.):

(8) Um das geheime Netzwerk der Moslem-Fanatiker zu zerstören, greifen die bedrohten Regime hart durch. Im Kampf gegen die Islamisten, klagt Amnesty International, habe sich die Menschenrechtslage etwa in Tunesien „wesentlich verschlechtert“. (SPIEGEL-005)

(9) Nach einer Attentatswelle gegen Kasernen und Polizeistationen drohte er, „weitere zehntausend" Islamisten in die Straflager zu stecken, um den Widerstand zu brechen. (SPIEGEL006)

(10) Mit dem Schlag gegen die Islamisten hat sich Frankreich offen in den Bruderkrieg eingemischt. Frankreich müsse die „algerischen Realitäten“ anerkennen, forderte der in Chicago residierende FIS-Führer Anwar Chaddam. (SPIEGEL-007)

Für unseren illustrativen Versuchsaufbau ist entscheidend - und wir werden uns hier nicht mit der Genese von Diskursen als „virtuellen Korpora“ länger aufhalten -, ob es sprachliche Muster, Konstruktionen gibt, die in einem thematisch zentrierten Diskurs („virtuelles Korpus“) um Schlagworte wie „Islamismus“ oder „Islam“ oder „Islamist“ (abgefragt wird nach Wortvorkommen *islam*) Hinweise darauf geben, wie sich die Neuprägung im Korpus beschreiben lässt. Anschließend gehen wir der Frage nach, ob diese beobachteten spezifischen sprachlichen Muster zur Erstellung eines „konkreten Korpus“ für eine diskursanalytische Untersuchung hinzugezogen werden können und welchen Bedingungen sie hierfür genügen müssen.

Für die kleine Beispielanalyse konzentrieren wir uns auf das SPIEGEL-Korpus (ca. 238 Millionen Textwörter) und führen eine Kollokationsanalyse durch: Wir rufen dafür den String ${ }^{*}$ islam $^{*}$ ab und ziehen Cluster (L11 ${ }^{*}$ islam $^{*}$ R11) aus dem Gesamtkorpus - diese Cluster bilden die Grundlage der Analyse. Auffällig ist, dass unabhängig vom Auftreten unterschiedlicher Schlagwörter seit den 1950er Jahren die Präpositionen für und gegen in den untersuchten Clustern häufig nachweisbar sind wie in den ausgewählten Beispielen (8)-(10): Mit einer Frequenz von 3328 (L1798 und R1530) steht für auf Platz 20 der Kollokate von *islam ${ }^{\star}$, gegen mit 1877 (L1097 und R790) auf Rang 33. 
Tab. 1: Gegen und für als Kollokate von X in Clustern L11 bis R11 in KERN- und ZEIT-Korpus.

\begin{tabular}{|c|c|c|c|c|c|c|c|c|c|c|}
\hline \multirow{3}{*}{$\begin{array}{c}\mathrm{X} \\
\text { Christentum }\end{array}$} & \multicolumn{2}{|c|}{$f_{x}$} & \multicolumn{4}{|c|}{ 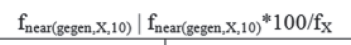 } & \multicolumn{4}{|c|}{$f_{\text {near(für, }, X, 10)} \mid f_{\text {near(für, }, X, 10)}{ }^{*} 100 / f_{X}$} \\
\hline & \multirow{2}{*}{$\begin{array}{r}\text { KERN } \\
3116\end{array}$} & \multirow{2}{*}{$\begin{array}{r}\text { ZEIT } \\
5696\end{array}$} & \multicolumn{2}{|c|}{ KERN } & \multicolumn{2}{|c|}{ ZEIT } & \multicolumn{2}{|c|}{ KERN } & \multicolumn{2}{|c|}{ ZEIT } \\
\hline & & & 106 & $3,40 \%$ & 113 & $1,98 \%$ & 260 & $8,34 \%$ & 434 & $7,62 \%$ \\
\hline Kapitalismus & 2699 & 12309 & 98 & $3,63 \%$ & 495 & $4,02 \%$ & 232 & $8,60 \%$ & 895 & $7,27 \%$ \\
\hline Kommunismus & 2088 & 11769 & 165 & $7,90 \%$ & 956 & $8,12 \%$ & 260 & $12,45 \%$ & 984 & $8,36 \%$ \\
\hline Islamismus & 2 & 577 & 0 & $\varnothing$ & 66 & $11,44 \%$ & 0 & $\varnothing$ & 49 & $8,49 \%$ \\
\hline [Islam $]$ & 915 & 9063 & 43 & $4,70 \%$ & 433 & $4,78 \%$ & 60 & $6,56 \%$ & 728 & $8,03 \%$ \\
\hline
\end{tabular}

\begin{tabular}{|l|r|r|r|r|r|r|r|r|r|r|}
\hline Freiheit & 15328 & 59126 & 449 & $2,92 \%$ & 1405 & $2,38 \%$ & 2019 & $13,17 \%$ & 7779 & $13,16 \%$ \\
\hline Atomkraft & 59 & 1442 & 9 & $15,24 \%$ & 114 & $7,91 \%$ & 8 & $13,56 \%$ & 167 & $11,58 \%$ \\
\hline Stift & 880 & 3992 & 8 & $0,65 \%$ & 26 & $0,65 \%$ & 46 & $5,23 \%$ & 226 & $5,56 \%$ \\
\hline Fenster & 13800 & 21480 & 253 & $0,74 \%$ & 160 & $0,74 \%$ & 301 & $2,18 \%$ & 849 & $3,95 \%$ \\
\hline
\end{tabular}

Das ist nicht ungewöhnlich für das Umfeld von Begriffen zur Bezeichnung einer Weltanschauung und andere umstrittene Begriffe: Ein Blick in die Vergleichskorpora (vgl. Tabelle 1) zeigt, ${ }^{7}$ dass trotz unterschiedlicher Qualität der Korpora Begriffe wie z. B. Christentum, Kommunismus und Kapitalismus ähnlich hochfrequent die Kollokate für und gegen ausweisen in Clustern selber Größe. Diese treten noch deutlicher heraus, wenn man die Belegzahlen ins Verhältnis setzt (Dreisatz; gerundet) - je höher der Wert, desto häufiger kommen die gesuchten Präpositionen um $\mathrm{X}$ in den Korpora vor.

Begriffe für Weltanschauungen dienen u.a. dazu, die Welt $\mathrm{zu}$ ordnen und zu sortieren, zu kategorisieren - so wundert es nicht, dass weltanschauliche Begriffe in der Nähe zu disjunkten Argumenten einer Argumentation in Clustern beobachtet werden oder selbst als solche aufgebaut werden und dementsprechend Präpositionen wie für und gegen in diesen Aussagezusammenhängen häufig auftreten. Merkmal disjunkter Argumentationen ist, dass sie die meist basale Dichotomie und damit das Nichtgesagte aktivieren und zugleich evaluieren. Ganz in diesem Sinne wird der Begriff des Islamismus erst in den 1980ern neu geprägt. Er wird als Begriff für eine Weltanschauung im direkten Umfeld disjunkter Argumentationen greifbar.

7 Verwendet werden hier das KERN- (ca. 100 Millionen Textwörter) und ZEIT-Korpus (ca. 106 Millionen Textwörter) beim Digitalen Wörterbuch der deutschen Sprache des 20. Jahrhunderts (DWDS). Die ermittelten Werte dienen lediglich der Illustration, weshalb auf die Angabe statistischer Maße verzichtet wird. Freiheit, Atomkraft, Stift und Fenster wurden als Kontrollbegriffe gewählt. Die Übersicht bietet weiter keinen Hinweis auf die Art und Weise des Gebrauchs von für und gegen - hier werden alle Gebrauchsarten abgebildet. 
Das von der Reagan-Administration für unseren Zusammenhang relvante „war against terrorism“ bzw. „war on terrorism“, 8 geprägt im Zuge des Anschlags von Beirut 1983, kann als typische Realisierung einer solchen Kollokation gesehen werden: Kriege und Debatten führt man für oder gegen etwas, für oder gegen jemanden (vgl. Lakoff / Johnson 1980). Die Präpositionen gegen und für, die Nominalphrasen im Akkusativ nehmen, haben in Aussagenkomplexen dieser Art, wie in den tabellarischen Übersichten gezeigt, eine herausgehobene Bedeutung; interessanterweise werden aber in den wenigsten funktionalen Grammatiken die Präpositionen gegen und für hinsichtlich ihrer Semantik ausführlich beschrieben: Eine Präposition ist ein „Wort, das Wörter zueinander in Beziehung setzt und ein bestimmtes (räumliches, zeitliches o.ä.) Verhältnis angibt; Verhältniswort (z. B. an, auf, bei, für, wegen, zu). “9 In der Duden-Grammatik (DUDEN 2009, IV, 607-622) werden Präpositionen zwar semantisch spezifiziert, eine genaue Angabe in Bezug auf für und gegen wie eine Aussage über typische Kollokate sucht man leider vergeblich. Eisenberg (2006, II, 190-198) stellt die Präpositionalgruppen (gegen und für als Kopf der PrGr) in drei syntaktischen Funktionen (Adverbial, Ergänzung [Objekt], Attribut) vor, legt den Schwerpunkt aber auf den „Kernbestand“ lokaler Präpositionen. Grammis unterscheidet nach temporalen, instrumentalen, finalen, direktionalen (gegen den Feind), lokalen und temporalen (gegen 12 Uhr; für einige Tage) Präpositionen. Die Bedeutung von Präpositionen wird - wie bei Eisenberg - anhand lokaler Präpositionen beschrieben. ${ }^{10}$

Wie könnte man das Verhältnis, dass die Präpositionen etablieren, aber semantisch genauer fassen, so dass sie für die diskurslinguistische Interpretation von Aussagekomplexen fruchtbar gemacht werden können? Die Konstruktionsgrammatik versucht Fragen wie diese zu beantworten. Die im Korpus im näheren Umfeld von *islam* auftretenden Präpositionen gegen und für weisen auf eine Spezifik von Aussagekomplexen hin, die positiv und negativ bewertete Sachverhalte gegeneinander abgrenzen und immer zugleich das Nichtgesagte mit evaluieren. Anders gesagt: Die Präpositionen für und gegen setzen nicht nur „Wörter in ein Verhältnis“ (also eine $\mathrm{NP}_{\mathrm{NOM}}$ und eine $\mathrm{NP}_{\mathrm{AKK}}$ ), sondern sie etablieren disjunkte Gegensätze, die evaluiert werden können. D.h., dass, wenn ein „Wort in ein Verhältnis“ zu einem anderen gesetzt wird (Kampf gegen Freiheit / Atomkraft), zugleich immer mitbedeutet wird, welche Option ausgeschlossen wird (Kampf

8 Silver 2010 und Chomsky 2003.

9 Duden-Rechtschreibung online, <http://www.duden.de/rechtschreibung/Praeposition>, Stand: 13.07.2014.

10 Grammis, <http://hypermedia.ids-mannheim.de/>, Stand: 13.07.2014. 
für Freiheit / Atomkraft). In der Logik bezeichnet man diesen Zusammenhang als „ausschließendes Oder“ (Disjunktion), als eine Kontravalenz.

Die besondere Leistung der Präpositionen ist, dass das jeweils andere nicht expliziert werden muss - konstruktionsgrammatisch ist daher fraglich, ob die Präpositionen für und gegen in dieser Verwendung eigenständig behandelt werden sollen. Vielmehr sollte dafür plädiert werden, hier zwei Präpositionen anzunehmen, die ein Verhältnis aus je verschiedener Perspektive in den Blick nehmen und damit zugleich immer Rückschlüsse auf Einstellungen der Sprecher zulassen. In der Konsequenz wären gegen und für in spezifischen Gebrauchszusammenhängen als Filler einer lexikalisch teilspezifizierten „Konstruktion der Kontravalenz“ [[für] / [gegen]+[NP]] zu analysieren, die je in zwei Weisen aktualisiert werden kann. Damit kann nicht nur die oben beschriebene Kontravalenz (11 und 12), sondern auch ein Sachverhalt perspektivenreich in Aussagekomplexe eingebunden werden (13 und 14):

(11) *für den Kampf gegen den Islamismus

(12) *gegen den Kampf gegen den Islamismus

(13) *für den Kampf für den Islamismus

(14) *gegen den Kampf für den Islamismus

Die maschinelle Sprachanalyse macht sprachliche Muster in einer langen Reihe sichtbar, die sich intuitiven, thematisch gesteuerten Zugriffen entziehen. Einige dieser Muster lassen sich als Konstruktionen beschreiben, z. B. als Realisierungen einer hier postulierten „Konstruktion der Kontravalenz“. Diese können in einem Konstruktionsnetzwerk, dem ,Konstruktikon', verortet werden, wie Abbildung 2 im Anschluss an die Beispiele (11)-(14) zeigt.

Die schematische Darstellung beruht auf dem Goldberg'schen Modell der internen Struktur der Konstruktion, das durch die semantischen Differenzierungsvorschläge der Polenz'schen Satzsemantik erweitert wurde. ${ }^{11}$ KÄMPFEN gibt den Prädikationsrahmen an, der zugleich die Konstruktionsbedeutung vorgibt, die „Konstruktion der Kontravalenz“ wird in diesen Rahmen eingebettet. Verschiedene Verben, wie im Beispiel kämpfen oder argumentieren, können in die

11 Vgl. dazu aber Lasch 2014 sowie Ziem / Lasch 2013, 110-142, bes. 122-129. Zu semantischen Rollen vgl. von Polenz 2008, 167-174, Ziem / Lasch 2013, 124-127 sowie Lasch 2014. - Grundsätzlich sei in diesem Zusammenhang explizit auch auf die Arbeiten des FrameNet hingewiesen. 


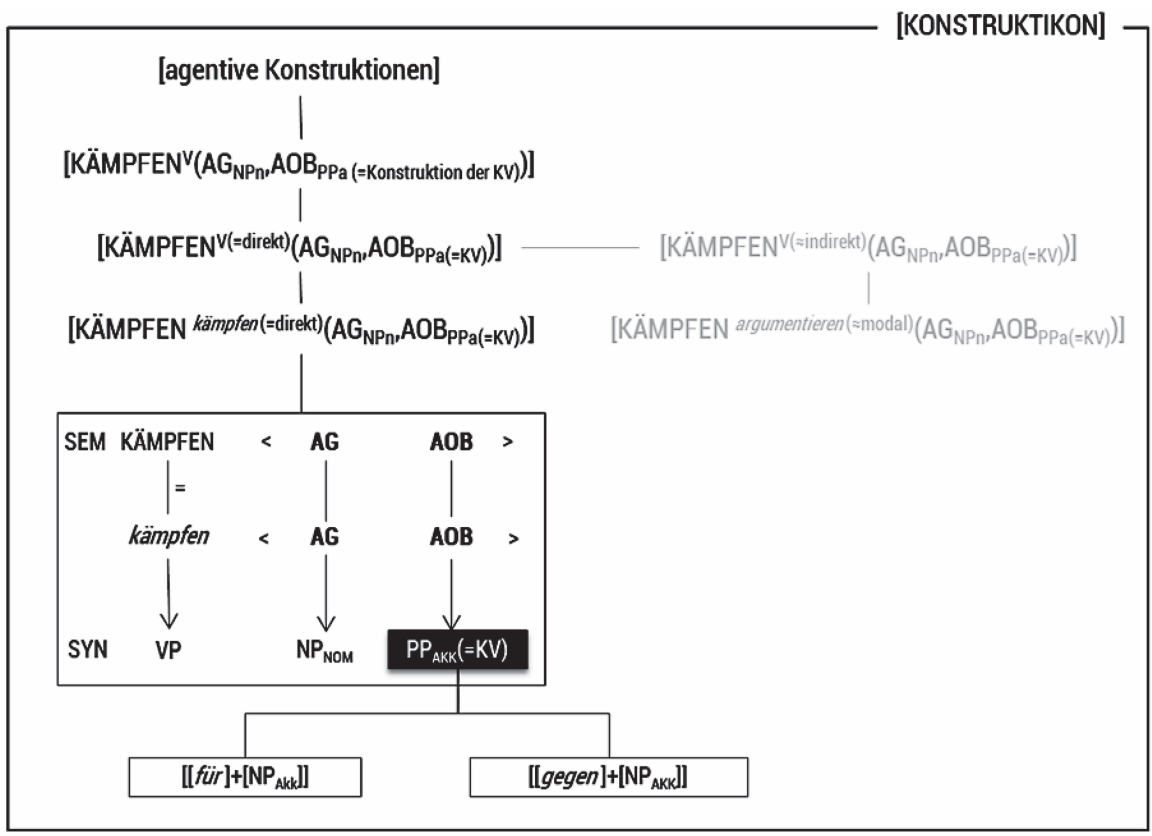

Abb. 2: „Konstruktion der Kontravalenz“ als Präpositionalphrase im Akkusativ eingebettet in die agentive Konstruktion KÄMPFEN (in direkter Lesart mit dem Verb kämpfen, indirekt, modal mit argumentieren).

Konstruktion eintreten - sie aktualisieren in direkter oder indirekter (modaler) Lesart die Konstruktionsbedeutung. ${ }^{12}$

Von diesen über die Semantik motivierten Konstruktionen können diskursanalytische Untersuchungen unmittelbar profitieren, denn unkonventionelle Kategorisierungsprozesse können formal beschrieben werden und z. B. für die Erläuterung von Argumentationsstrukturen fruchtbar gemacht werden. So wäre nun bspw. zu fragen, welche Diskursposition ein Diskursakteur vertritt, wenn er eine Variante der „Konstruktion der Kontravalenz“ gebraucht? Wie zu sehen war, ist es die Leistung der Konstruktion, Bezeichnetes als disjunktes Argument in einer Argumentation zu etablieren und zu stabilisieren, was im exemplarisch

12 Die Leistungsfähigkeit dieses Ansatzes z.B. gegenüber valenzgrammatischen Annahmen, die evtl. einen Eintrag wie kämpfen für / gegen oder argumentieren für / gegen postulieren würden, wird besonders deutlich, wenn man auch andere Verben in Bezug auf die Frage prüft, ob und wie sie in (indirekter Lesart in) diesen Prädikationsrahmen eingebettet werden können: ${ }^{\star}$ Sie fahren / backen / spenden für / gegen den Frieden. 
skizzierten Prädikationsrahmen KÄMPFEN besonders deutlich wird. Das hat für den diskursiv verhandelten Begriff Islamismus, selbst wenn er nicht Teil der Konstruktion ist, sondern in unmittelbarer Nähe steht, erhebliche Konsequenzen: Er wird nicht nur assertativ benannt, sondern primär als disjunktes Argument deklariert bzw. erscheint in direktem Umfeld einer solchen Festlegung. Ergebnis sind starke (und teils sehr beharrliche) Dichotomien, die anhand oberflächlicher sprachlicher Strukturen Rückschlüsse auf das in Sprache sedimentierte Wissen unserer Diskursgemeinschaft gestatten. Aber damit nicht genug: Bei der „Konstruktion der Kontravalenz“ handelt es sich nicht um ein Phänomen der Sprachoberfläche, sondern um einen deep surface frame, der unbewusst unser Denken lenkt - es ist nicht allein das „Schlüsselwort“ wie Islamismus oder Kommunismus, das auf der sprachlichen Oberfläche einen Frame etabliert, es ist die „Konstruktion der Kontravalenz“, die maßgeblich für die Modellierung eines Gegensatzes verantwortlich ist und dabei das Besondere leistet, dass nicht beide Gegensätze, sondern nur eines der disjunkten Argumente aufgerufen werden muss. Wie bereits zu vermuten, ist es also weniger die Schlagwortanalyse, die uns hier voranbringt (und die basal war für die Etablierung eines „konkreten Korpus“), sondern die maschinelle Analyse „,virtueller Korpora“ auch anderer thematischer Zentrierung, die ein sprachliches Muster, die „Konstruktion der Kontravalenz“, sichtbar werden ließ, die sich in unserem „konkreten Korpus“ zum „Islamismus“ beobachten ließ und keineswegs nur für dieses typisch ist. Als deep surface frame ist sie hier schon viel länger aktiv, noch weit bevor die Neuprägung des Begriffs Islamismus in den 1980ern erfolgt, wie etwa die Beispiele (15) und (16) mit dem attributiven Adjektiv islamisch und dem Nomen Islam belegen:

(15) Nicht daß man im Foreign Office etwas gegen islamische Frömmigkeit hat. Ihr Verdacht richtet sich gegen die Abstecher, die die frommen Pilger gewöhnlich nach Riadh zu machen pflegen. (SPIEGEL-008)

(16) Der „Große Brockhaus“ bezeichnet heute die Lehre vom „Dschihad“, vom heiligen Krieg des Islams gegen die Nicht-Mohammedaner, die dem Gesetz des Propheten unterworfen werden sollen, als eine für die moderne Zeit nur noch theoretische Angelegenheit von allein historischer Bedeutung. (SPIEGEL-009)

Die Neuprägung des Begriffs Islamismus ist nicht der Anfang, sondern Höhepunkt und Ergebnis einer sprachlichen Entwicklung, in der der Begriff Islam zunehmend für eine Weltanschauung verwendet wird, die Bewertungen unterzogen wird und deshalb im direkten Umfeld disjunkter Argumentationen aufscheint. 


\section{Fazit}

Die sprachhistorische Forschung kann zwar noch nicht auf Korpora zurückgreifen, die quantitative Untersuchungen in der historischen Tiefe erlauben. Aber sie kann, diskurslinguistisch motiviert, Beobachtungen an „konkreten Korpora“ des 20. Jahrhunderts in andere „virtuelle Korpora“ spiegeln und sprachliche Muster konstruktionsgrammatisch erarbeiten, die Interpretationen in einem thematisch zentrierten konkreten Korpus wesentlich ergänzen können. Mithilfe der Konstruktionsgrammatik und ihrem zentralen Anliegen, semantische Potentiale sprachlicher Muster, wenn möglich, zu Konstruktionen zu systematisieren, kann der Brückenschlag zwischen Diskurslinguistik und Korpuslinguistik gelingen. Für sprachhistorische Arbeiten ist dies äußerst reizvoll: Konstruktionen wie die hier beschriebene „Konstruktion der Kontravalenz“ erfreuen sich wie viele grammatikalisierte sprachliche Phänomene erstaunlicher Stabilität, so dass sie in diachronen Korpora leichter identifiziert werden können und damit - vor allem in Kollostruktionsanalysen - systematische Zugriffe auf sprachhistorisch relevante Gegenstände wesentlich erleichtern können.

Der Beitrag versuchte darüber hinaus $\mathrm{zu}$ illustrieren, dass sich diskurslinguistisch motivierte Studien nicht mehr ausschließlich auf den etablierten Diskursbegriff verlassen können, der den Diskurs mit dem „virtuellen Korpus“ gleichsetzt. Dies würde keine Rückschlüsse auf die Verteilung und den Gebrauch grammatischer Phänomene wie der „Konstruktion der Kontravalenz“ erlauben. Stichprobenartige Abfragen in thematisch nicht zentrierten Korpora (DWDS) zeigten, dass es schlicht unwahrscheinlich ist, dass die „Konstruktion der Kontravalenz“ für das hier im Mittelpunkt stehende „virtuelle Korpus“ zum „Islamismus“ typisch ist.

\section{Literatur}

\subsection{Quellen}

\subsubsection{Korpusbelege}

Hazzi, Joseph von (1822), Ueber den Islamismus, das Türkenthum, dann die Sache der Griechen und Europens Pflichten dabei, München.

Krug, Wilhelm Traugott (Hrsg.) (1833), Allgemeines Handwörterbuch der philosophischen Wissenschaften nebst ihrer Literatur und Geschichte. Nach dem heutigen Standpuncte der Wissenschaft, Band 2., 2. bearb., verm. und verb. Aufl., Leipzig. 
Oelsner, Konrad Engelbert (1810), Mohamad. Darstellung des Einflusses seiner Glaubenslehre auf die Völker des Mittelalters. Eine Preisschrift, welche von dem französischen National=Institut der Wissenschaften am 7. July 1809 gekrönt wurde, Frankfurt am Main.

Schmid, A. J. (1815), „Völkerkunde. Bewohner der Barbarey“, in: Friedensblätter. Eine Zeitschrift für Leben, Literatur und Kunst, 120, 477-480.

SPIEGEL-001: „Schweiz-Report“, in: SPIEGEL 32 / 1971, http://goo.gl/daf7K1 (Stand: 13.07.2014)

SPIEGEL-002: „Algerien: Abschied von der Revolution“, in: SPIEGEL 12 / 1989, http://goo.gl/ e7DCHv (Stand: 13.07.2014)

SPIEGEL-003: „Moralisches Urteil“, in: SPIEGEL 17 / 1999, http://goo.gl/ZJ5BM4 (Stand: 13.07.2014)

SPIEGEL-004: „Rache für den Kalifen“, in: SPIEGEL 13 / 1999, http://goo.gl/GEwV88 (Stand: 13.07.2014)

SPIEGEL-005: „Spiel mit dem Nilwasser“, in: SPIEGEL 32 / 1991, http://goo.gl/M6hyc9 (Stand: 13.07.2014)

SPIEGEL-006: „Nur noch der Abgrund“, in: SPIEGEL 28 / 1992, http://goo.gl/DwfxDr (Stand: 13.07.2014)

SPIEGEL-007: „In der Falle“, in: SPIEGEL 46 / 1993 http://goo.gl/KJuIRI (Stand: 13.07.2014)

SPIEGEL-008: „Auf einem Esel reitend“, in: SPIEGEL 25 / 1952 http://goo.gl/QRZxa3 (Stand: 13.07.2014)

SPIEGEL-009: „Keine andere Wahl“, in: SPIEGEL 52 / 1952, http://goo.gl/cOCUJk (Stand: 13.07.2014)

\subsubsection{Vergleichskorpora und weitere Datenquellen}

DWDS KERN- und ZEIT-Korpus, http://dwds.de/ (Stand: 13.07.2014)

Duden-Rechtschreibung online, http://www.duden.de/rechtschreibung/Praeposition, (Stand: 13.07.2014)

Grammis, http://hypermedia.ids-mannheim.de/ (Stand: 13.07.2014)

Islamism, Islamismus in den englisch- und deutschsprachigen Korpora bei Google Books,

Suchabfrage im nGram Viewer, http://goo.gl/17Jxqv (Stand: 13.07.2014)

FrameNet, https://framenet.icsi.berkeley.edu/fndrupal/ (Stand: 13.07.2014)

„Report of the DOD Commission on Beirut International Airport Terrorist Act, October 23, 1983“, http://goo.gl/vK87GO (Stand: 13.07.2014).

\subsection{Forschungsliteratur}

Aust, Stefan (2008), Der Baader Meinhof Komplex, völl. überarb. und erg. Neuausgabe, Hamburg.

Boas, Hans C. (2014), „Zur Architektur einer konstruktionsbasierten Grammatik des Deutschen“, in: Alexander Lasch / Alexander Ziem (Hrsg.), Grammatik als Netzwerk von Konstruktionen: Sprachwissen im Fokus der Konstruktionsgrammatik, (Sprache und Wissen, 15), Berlin / Boston, 37-63. 
Braudel, Fernand (1977), „Geschichte und Sozialwissenschaften - Die longue durée“, in: Claudia Honegger (Hrsg.), Schrift und Materie der Geschichte. Vorschläge zur systematischen Aneignung historischer Prozesse, Frankfurt am Main, 47-85.

Bücker, Jörg (2014), „Konstruktionen und Konstruktionscluster: das Beispiel der Zirkumposition von XP her im gesprochenen Deutsch“, in: Alexander Lasch / Alexander Ziem (Hrsg.), Grammatik als Netzwerk von Konstruktionen: Sprachwissen im Fokus der Konstruktionsgrammatik, (Sprache und Wissen, 15), Berlin / Boston, 117-135.

Busse, Dietrich (1987), Historische Semantik. Analyse eines Programms, Stuttgart.

Busse, Dietrich (1997), „Das Eigene und das Fremde. Annotationen zu Funktion und Wirkung einer diskurssemantischen Grundfigur“, in: Matthias Jung / Martin Wengeler / Karin Böke (Hrsg.), Die Sprache des Migrationsdiskurses. Das Reden über ,Ausländer ' in Medien, Politik und Alltag, Opladen, 17-35.

Busse, Dietrich (2000), „Historische Diskurssemantik. Ein linguistischer Beitrag zur Analyse gesellschaftlichen Wissens", in: Sprache und Literatur in Wissenschaft und Unterricht, 31 / 86, 39-53.

Busse, Dietrich (2007), „Diskurslinguistik als Kontextualisierung - Sprachwissenschaftliche Überlegungen zur Analyse gesellschaftlichen Wissens“, in: Ingo H. Warnke (Hrsg.), Diskurslinguistik nach Foucault. Theorie und Gegenstände, (Linguistik - Impulse \& Tendenzen 25), Berlin / New York, 81-105.

Busse, Dietrich (2008), „Diskurslinguistik als Epistemologie. Das verstehensrelevante Wissen als Gegenstand linguistischer Forschung“, in: Ingo H. Warnke / Jürgen Spitzmüller (Hrsg.), Methoden der Diskurslinguistik. Sprachwissenschaftliche Zugänge zur transtextuellen Ebene, (Linguistik - Impulse \& Tendenzen, 31), Berlin / New York, 57-87.

Busse, Dietrich (2012), Frame-Semantik. Ein Kompendium, Berlin / Boston.

Busse, Dietrich / Wolfgang Teubert (2013), „Ist Diskurs ein sprachwissenschaftliches Objekt? Zur Methodenfrage der historischen Semantik“ (unveränd. Nachdruck von 1994), in: Dietrich Busse / Wolfgang Teubert (Hrsg.), Linguistische Diskursanalyse: Neue Perspektiven, Wiesbaden, 13-30.

Chomsky, Noam (2003), „Wars of Terror“, in: New Political Science, http://goo.gl/fyxT7f (Stand: 13.07.2014).

Coseriu, Eugeniu (1974), Synchronie, Diachronie und Geschichte: Das Problem des Sprachwandels, München.

DUDEN (2009, IV) = Duden (2009), Die Grammatik, 8. Aufl., Mannheim u.a.

Eisenberg, Peter (2006, II), Grundriss der deutschen Grammatik, Band 2: Der Satz, 3., durchges. Aufl., Stuttgart / Weimar.

Feilke, Helmut (1996), Sprache als soziale Gestalt. Ausdruck, Prägung und die Ordnung der sprachlichen Typik, Frankfurt am Main.

Felder, Ekkehard / Marcus Müller / Friedemann Vogel (2012), „Korpuspragmatik - Paradigma zwischen Handlung, Gesellschaft und Kognition“, in: Ekkehard Felder / Marcus Müller / Friedemann Vogel (Hrsg.), Korpuspragmatik. Thematische Korpora als Basis diskurslinguistischer Analysen, (Linguistik - Impulse \& Tendenzen, 44), Berlin / New York, 2-28.

Goldberg, Adele E. (1995), Constructions: A Construction Grammar Approach to Argument Structure, Chicago.

Goldberg, Adele E. (2003), „Constructions: a new theoretical approach to language“, Trends in Cognitive Sciences, 7 / 5, 219-224.

Goldberg, Adele E. (2006), Constructions at Work. The Nature of Generalization in Language, Oxford. 
Hermanns, Fritz (1995), „Sprachgeschichte als Mentalitätsgeschichte. Überlegungen zu Sinn und Form und Gegenstand historischer Semantik“, in: Andreas Gardt / Klaus J. Mattheier / Oskar Reichmann (Hrsg.), Sprachgeschichte des Neuhochdeutschen. Gegenstände, Methoden, Theorien, (RGL 156), Tübingen, 69-99.

Honegger, Claudia (1977), „Geschichte im Entstehen. Notizen zum Werdegang der ,Annales““, in: Claudia Honegger (Hrsg.), Schrift und Materie der Geschichte. Vorschläge zur systematischen Aneignung historischer Prozesse, Frankfurt am Main, 7-44.

Lakoff, George (1987), Women, Fire, and dangerous Things. What Categories Reveal about the Mind, Chicago.

Lakoff, George (2002), Moral Politics: How Liberals and Conversvatives Think, 2., stark akt. Aufl., Chicago.

Lakoff, George / Eva Elisabeth Wehling (2009), Auf leisen Sohlen ins Gehirn. Politische Sprache und ihre heimliche Macht, 2., akt. Aufl., Heidelberg.

Lakoff, George / Mark Johnson (1980), Metaphors We Live By, Chicago.

Lasch, Alexander (2005), Beschreibungen des Lebens in der Zeit. Zur Kommunikation biographischer Texte in den pietistischen Gemeinschaften der Herrnhuter Brüdergemeine und der Dresdner Diakonissenschwesternschaft im 19. Jahrhundert, Münster.

Lasch, Alexander (2013), „Sind serielle Texte ein Gegenstand linguistischer Diskursanalyse? Zu diskursbestätigenden und diskursverändernden ,Lebensbeschreibungen ' in rituellen Kontexten“, in: Dietrich Busse / Wolfgang Teubert (Hrsg.), Linguistische Diskursanalyse: neue Perspektiven, Wiesbaden, 381-399.

Lasch, Alexander (2014), „Das Fenster wirkt geschlossen - Überlegungen zu nonagentiven Konstruktionen des Deutschen aus konstruktionsgrammatischer Perspektive“, in Alexander Lasch / Alexander Ziem (Hrsg.), Grammatik als Netzwerk von Konstruktionen: Sprachwissen im Fokus der Konstruktionsgrammatik, (Sprache und Wissen, 15), Berlin / Boston, 65-95.

Lasch, Alexander / Alexander Ziem (Hrsg.) (2014), Grammatik als Netzwerk von Konstruktionen: Sprachwissen im Fokus der Konstruktionsgrammatik, (Sprache und Wissen, 15), Berlin / Boston.

Mattheier, Klaus J. (1995), „Sprachgeschichte des Deutschen: Desiderate und Perspektiven“, in: Andreas Gardt / Klaus J. Mattheier / Oskar Reichmann (Hrsg.), Sprachgeschichte des Neuhochdeutschen. Gegenstände, Methoden, Theorien, (RGL 156), Tübingen, 1-18.

Polenz, Peter von (2008), Deutsche Satzsemantik. Grundbegriffe des Zwischen-den-Zeilen-Lesens, 3., unveränd. Aufl. der Ausgabe von 1985, Berlin / New York.

Rostila, Jouni (2014), „Inventarisierung als Grammatikalisierung: produktive Präpositionalobjekte und andere grammatikalisierte Linking-Muster“, in: Alexander Lasch / Alexander Ziem (Hrsg.), Grammatik als Netzwerk von Konstruktionen: Sprachwissen im Fokus der Konstruktionsgrammatik, (Sprache und Wissen, 15), Berlin / Boston, 97-116.

Scharloth, Joachim / David Eugster / Noah Bubenhofer (2013), „Das Wuchern der Rhizome. Linguistische Diskursanalyse und Data-driven Turn“, in: Dietrich Busse / Wolfgang Teubert (Hrsg.), Linguistische Diskursanalyse: neue Perspektiven, Wiesbaden, 345-380.

Schlieben-Lange, Brigitte (1983), „Geschichte der Sprachwissenschaft und Geschichte der Sprachen“, in: Bernhard Cerquiglini / Hans Ulrich Gumbrecht (Hrsg.), Der Diskurs der Literatur- und Sprachhistorie. Wissenschaftsgeschichte als Innovationsvorgabe, Frankfurt am Main, 464-491.

Silver, Alexandra (2010), „How America Became a Surveillance State“, in: Time Magazine vom 18. Mai 2010, http://goo.gl/uc0Ju7 (Stand: 13.07.2014). 
Spitzmüller, Jürgen / Ingo H. Warnke (2011), Diskurslinguistik. Eine Einführung in Theorien und Methoden der transtextuellen Sprachanalyse, Berlin / New York.

Ziem, Alexander (2008), Frames und sprachliches Wissen: kognitive Aspekte der semantischen Kompetenz, (Sprache und Wissen, 2), Berlin / New York.

Ziem, Alexander (2014a), „Konstruktionsgrammatische Konzepte eines Konstruktikons“, in: Alexander Lasch / Alexander Ziem (Hrsg.), Grammatik als Netzwerk von Konstruktionen: Sprachwissen im Fokus der Konstruktionsgrammatik, (Sprache und Wissen, 15), Berlin / Boston, 15-34.

Ziem, Alexander (2014b), „Von der Kasusgrammatik zum FrameNet: Frames, Konstruktionen und die Idee eines Konstruktikons“, in: Alexander Lasch / Alexander Ziem (Hrsg.), Grammatik als Netzwerk von Konstruktionen: Sprachwissen im Fokus der Konstruktionsgrammatik, (Sprache und Wissen, 15), Berlin / Boston, 263-290.

Ziem, Alexander / Alexander Lasch (2013), Konstruktionsgrammatik. Konzepte und Grundlagen gebrauchsbasierter Ansätze, (Germanistische Arbeitshefte, 44), Berlin / Boston. 